\title{
Does a population-based multi-factorial lifestyle intervention increase social inequality in smoking? The Inter99 study
}

\author{
Charlotta Pisinger $^{1^{*}}$, Mette Aadahl ${ }^{1}$, Ulla Toft $^{1}$, Anne Helms Andreasen ${ }^{1}$, \\ Torben Jørgensen ${ }^{1,2,3}$ \\ ${ }^{1}$ Research Centre for Prevention and Health, Glostrup University Hospital, The Capital Region of Denmark, Copenhagen, Denmark; \\ ${ }^{*}$ Corresponding Author: charlotta.pisinger@regionh.dk \\ ${ }^{2}$ Faculty of Health Science, Copenhagen University, Copenhagen, Denmark \\ ${ }^{3}$ Faculty of Medicine, University of Aalborg, Aalborg, Denmark
}

Received 20 February 2012; revised 28 March 2012; accepted 16 April 2012

\section{ABSTRACT}

Introduction: The social inequality in smoking in the Western countries has been increasing. It has been suggested that the most important strategy to reduce health inequalities related to socio economic status (SEP) is to promote smoking cessation in persons with low SEP. One could fear that a smoking cessation intervention might benefit smokers with high SEP more, and thereby increase the social inequality in smoking. We wanted to investigate whether the effect differed across SEP. Methods: The study was an individual multi-factorial lifestyle intervention study with a control group, Inter99 (1999-2006), Copenhagen, Denmark. We included 1991 dailysmokers with self-reported information on education in the intervention group, and 1135 in the control group. Smokers in the intervention group were repeatedly offered individual lifestyle-counselling and group-based smoking cessation. We used generalised linear mixed models under the assumption of missing at random, including interaction term between intervention effect and SEP. Results: The gap in self-reported abstinence rates increased over time between persons with high and low education. Probability of abstinence was significantly higher in the intervention group than in the control group, at all follow-up-visits but the effect of the intervention changed over time. The differences in quit-rates across educational groups were not significantly different in the intervention than in the control group at any time. Conclusion: In this randomised population-based intervention study we found that smokers across all educational levels benefited from the anti-smoking interven- tion, and that the intervention did not increase the social inequality in smoking, as one could have feared.

Keywords: Smoking Cessation; Smoking; Socioeconomic Factors; Intervention Studies; Inter99 Study

\section{INTRODUCTION}

Persons with low socioeconomic position (SEP) often have difficult living conditions making them susceptible to disease $[1,2]$ and there is a strong association between population health and income inequality levels [3]. However, a recently published study found that smoking is a greater source of health inequality than social position [4]. The study suggests that the most important strategy to reduce health inequalities related to social position is to promote smoking cessation in persons with low social position. The social inequality in smoking in the Western countries has been increasing in the last decades [5-9] and an English report (Securing good health for the whole population) concluded "We do not know what messages and interventions work to get lower socioeconomic groups to stop smoking" [10]. When planning strategies/interventions to reduce social inequalities in health, evidence of the effect of the strategy is needed before implementing it. In the worst case a strategy could have the opposite effect, and increase the social inequality in smoking.

A large Danish population-based multi-factorial intervention study, the Inter99 study, used a proactive approach and an intensive, repeated lifestyle intervention over five years, resulting in significantly higher quit rates than in the control group [11]. The question is, whether smokers from all socioeconomic groups did benefit from 
the intervention, or whether the intervention only increased abstinence rates in smokers with high social position, and thereby increased the social inequality in smoking and health.

We have already shown that the intervention did not increase the social inequality in physical activity or diet, and that it may even have decreased or hindered further widening of the social inequalities in health due to unhealthy dietary habits among socially disadvantaged individuals [12,13].

The aim of this paper is to investigate the effect of a population-based multi-factorial intervention on smoking-across socioeconomic groups. Our hypothesis is that the intervention did not increase the social inequality in smoking.

\section{METHODS}

Inter99 is a population-based intervention study, initiated in March 1999 and ended in April 2006. The study design is described in detail elsewhere [14,15]. The study was performed at the Research Centre for Prevention and Health, Glostrup University Hospital, Copenhagen, Denmark, and was approved by The Copenhagen County Ethical Committee (KA 98155) and the National Board of Health and registered in the Clinical Trials.gov (NCT00289237). The aim of the study was to prevent cardiovascular disease by non-pharmacological intervention. We screened a general population for high risk of ischemic heart disease (IHD) and offered assistance to a healthier lifestyle to those at high risk. All smokers were considered to be at high risk of IHD.

\section{Study population}

The study population consisted of all 61,301 individuals aged $30-60$ years living in 11 municipalities in the suburbs south-west of Copenhagen City. An age- and sex-stratified random sample of 13,016 individuals was drawn from the Civil Registration by computer generated random numbers. Before invitation the sample was prerandomised into a high-intensity intervention $\mathrm{A}(\mathrm{n}=$ $11,708)$ and a low-intensity intervention group $\mathrm{B}(\mathrm{n}=$ 1308). Eighty-two persons had died or could not be traced. Of the remaining 12,934, a total of $6906(53.4 \%)$ participated in the study. Of these, 122 were excluded because of alcoholism, drug abuse or linguistic barriers, leaving 6784. Because of the small sample size and in order to keep the design simple the low-intensity intervention group is not included in the present study. From the remaining 48,285 individuals, a random sample of 5264 individuals was drawn (control group C) (Figure 1). A total of 3324 responded.

Participants included in this paper

This paper is based on daily smokers with information on occupational education at baseline in the high inten- sity intervention group $A(N=1991)$ and in the control group $\mathrm{C}(\mathrm{N}=1135)$ (Figure 1). In the intervention group, at five year follow-up, we additionally sent short questionnaires to non-attendants to gather information on smoking status. This information has been included in analyses.

\section{Intervention in group $A$}

At baseline, participants in the intervention group completed questionnaires and had an extensive health examination. They were categorised as high risk individuals if they had a high absolute risk of IHD, were daily smoker or obese, had hypertension, hypercholesterolemia, diabetes or impaired glucose tolerance. A total of $60 \%$ were categorised as high-risk individuals. All daily smokers were regarded to have high risk of IHD.

Based on the personal risk estimate, each participant had individual lifestyle counselling. All smokers were encouraged to quit (described in details elsewhere) [11, $15,16]$. In addition to the individualised lifestyle counselling, high-risk individuals (including all smokers) in the intervention group were offered lifestyle counselling in groups. At baseline $26.5 \%$ of the daily smokers accepted participation in smoking cessation groups [17]. Other smokers preferred to try to quit without further assistance than the lifestyle counselling.

There was no difference in SEP among smokers who accepted to participate in smoking cessation groups. An intensive non-pharmacological (behavioural) approach was combined with a pharmacological approach.

All smokers in the intervention were re-invited to a follow-up visit with lifestyle counselling after one, three and five years and offered participation in smoking cessation groups at one- and three-year follow-up.

Control group $C$

Participants in the control group only received questionnaires at baseline, one-, three- and five-year followup (Figure 1). Participants were not aware of an ongoing lifestyle intervention, as we wanted to observe natural temporal lifestyle-changes in an unexposed population.

\section{Outcome measure}

Self-reported point abstinence: those who reported to have quit at the time of a follow-up visit-independently of smoking status at other follow-up visits. Thus, a person could be point abstinent at one-year follow-up but have started smoking again at five-year follow-up. Abstinence was validated in the intervention group, but not in the control group [18]. Thus, we can only use selfreported abstinence in this paper.

\section{Measure of socioeconomic position}

Socioeconomic position: was defined by self-reported length of occupational education after finishing basic school education (e.g. unskilled worker $=0$ years, green keeper assistant $=1.5$ year, carpenter $=3.5$ years, teacher $=4$ years, medical doctor $=6$ years $)$. High education 


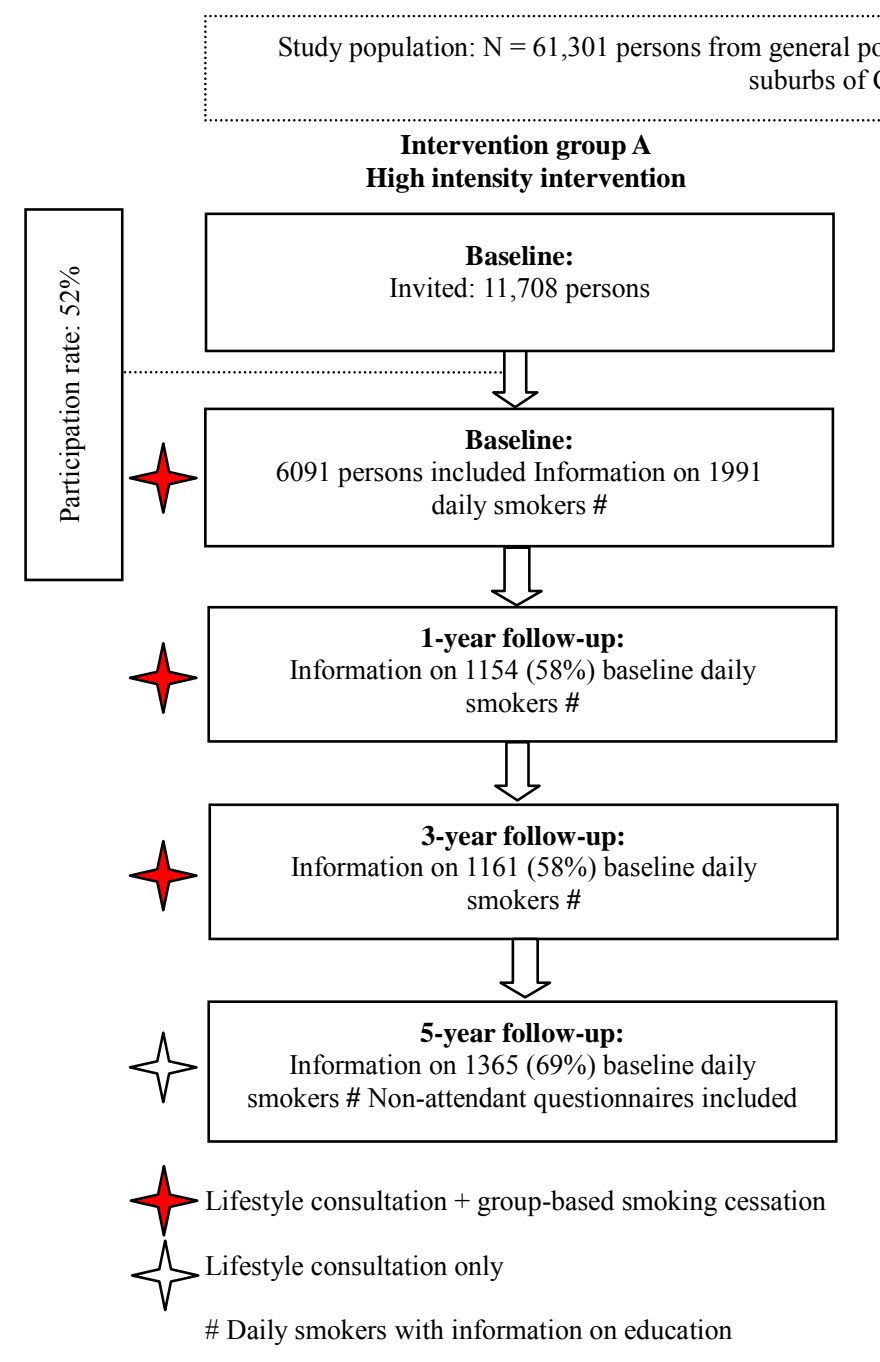

Control group C

No intervention (background population)

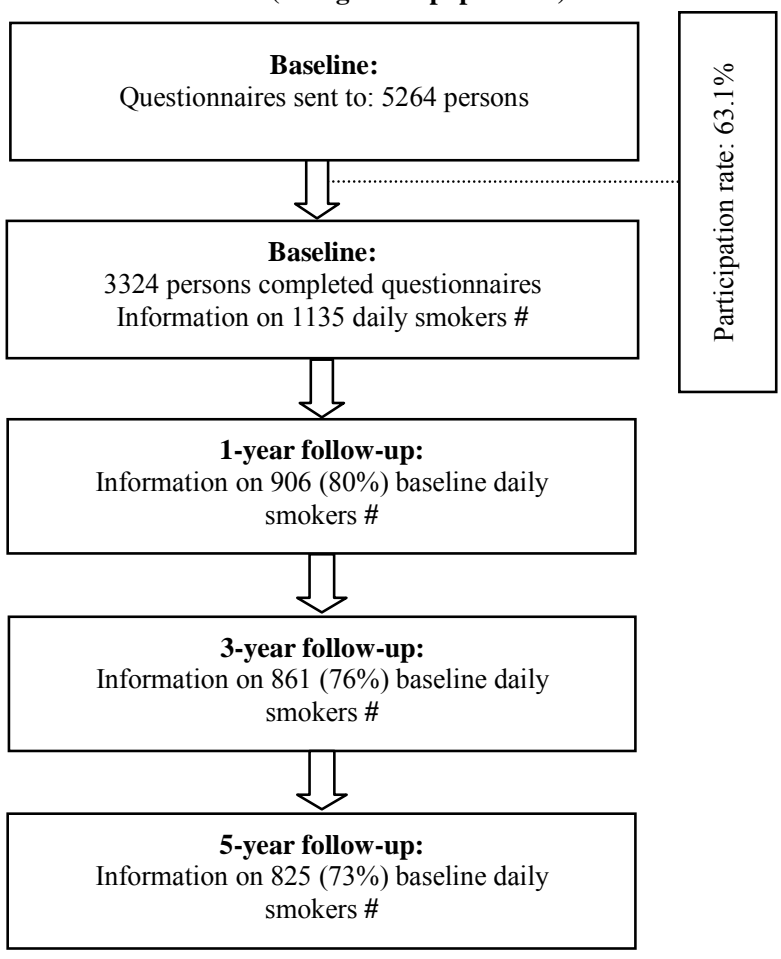

Cumulated number of dead + emigrated in the whole study

\begin{tabular}{|c|c|c|}
\hline & $\mathrm{A}$ & $\mathrm{C}$ \\
\hline 1-year & 13 & 16 \\
\hline 3-years & 27 & 30 \\
\hline 5-years & 55 & 43 \\
\hline
\end{tabular}

Figure 1. Flowchart of daily smokers at baseline with information on length of education in the Inter99 study (1999-2006), Copenhagen, Denmark.

(academic education) was more than four years, medium education one to four years, and low education less than one year. Occupational education is found to be significantly associated with chronic disease [19], low health knowledge, high un-employment rate, bad self-reported health and unhealthy lifestyle.

Co-variate measures (baseline)

Tobacco consumption: was measured as grams of tobacco $($ a cigarette $=$ one gram, a cheroot $=$ three grams, and a cigar = five grams; pipe tobacco was assessed in grams). A total of $94.4 \%$ of all daily smokers were cigarette smokers.

Motivation to quit: smokers were categorised in motivational stages using a simplified typology of Prochaska and Di Clemente [20,21]. (Preparation stage: "planning to quit within one month". Contemplation stage: "plan- ning to quit within the next six months". Precontemplation stage: "not planning to quit").

Dietary quality score: a three-class variable (healthy, average, un-healthy) was generated for each of the four food-groups/nutrients (fish, vegetable, fruit and fat) from a 52-item food frequency questionnaire (reference period: one week). The score has been validated [22]. Physical activity was based on self-reported commuting and leisure time physical activities and measured as minutes per week Recommended $=$ minimum 4 hours per week [23].

Alcohol consumption was assessed as mean self-reported consumption of units of beer/strong beer, wine and spirits per week. One beer or one glass of wine or four cl. of spirits equals one unit (approximately 12 gram alcohol). Strong beer equals 1.5 units. The Danish National Board of Health recommended less than 15 units 
of alcohol weekly for women and 22 for men.

\section{Statistical Analyses}

Data processing was done with the SPSS 15.0 software (SPSS Inc., Chicago, IL, USA).

When looking at baseline differences between intervention and control group, categorical data were tested by Persons Chi-square test and continuous data were tested by T-tests, after confirming that there was homogeneity of variances. As number of quit attempts and pack-years were skewed we tested differences by MannWhitney U-test.

In order to identify predictors of drop-out we tested the following factors in logistic regression analyses: age, sex, group, employment status, occupational education, living with partner, nationality, physical activity, diet, alcohol, self-reported physical health (measured by SF-12), motivation to quit and tobacco consumption. Young age, intervention group, low education, being employed, worse self-reported physical health, and higher tobacco consumption were found to be predictors of dropout at five-year follow-up. The logistic regression models were controlled by the Hosmer-Lemeshow goodnessof-fit test.

As we supposed that missing values were not randomly distributed across all observations but randomly distributed within subsamples (e.g. age or employment status) a generalised linear mixed model was used for analyses. This model uses information for all available measurements for each individual and is assumed to give valid inference under a missing at random (MAR) assumption. These analyses were performed in SAS version 9.2 (TS2M0). We used the generalised linear mixed model, with self-reported abstinence as outcome, subject as a random effect (to account for the repeated measurements within each person) and time of measurement, intervention/control-group and occupational education as fixed effect. The model was adjusted for sex, age, motivation to quit, tobacco consumption, diet, employment status and self-reported physical health as these factors were either 1) differently distributed across interventionand control group at baseline and/or 2) differently distributed at baseline between participants and drop-outs at one-, three- and five-years follow-up. In order to determine the impact of the intervention on self-reported abstinence from smoking across socioeconomic position, an interaction term between intervention and occupational education was included. Thereafter, an interaction term between intervention and time was included, in order to determine the effects of the intervention on selfreported abstinence from smoking across time. When exploring whether there was an impact of time on the effect of the intervention across socioeconomic position, a three-way interaction term comprising level of education, intervention group and time was included (as well as the interaction term between occupational education and time). Interactions were assessed on the multiplicative scale.

Level of significance was set to $5 \%$ in all analyses.

\section{RESULTS}

The significant differences between daily smokers in the intervention group and the control group at baseline were lower age, healthier diet, a higher motivation to quit, and a higher employment rate in the intervention group (Table 1). There were no differences in occupational education or number of school years.

We found higher abstinence rates in the intervention group than in the control group (Figure 2). The multivariate analyses showed a significant effect of the intervention, but the effect of the intervention changed over time $(p=0.035)$. Probability of self-reported abstinence was higher in the intervention group than in the control group, both at one- $(\mathrm{OR}=3.14 ; 95 \% \mathrm{CI}=2.1-4.7)$, three- $(\mathrm{OR}=1.72 ; 95 \% \mathrm{CI}=1.3-2.4)$ and five-year follow-up $(\mathrm{OR}=2.45 ; 95 \% \mathrm{CI}=1.8-3.3)$.

There was an educational trend, with highest self-reported abstinence rates in persons with high education, intermediate in persons with medium education, and lowest in those with low education (Figure 2). The gap in abstinence seemed to increase over time between persons with high and low education. The intervention did not widen the educational gap. It actually seemed to narrow the gap a little over time. At five-year follow-up, smokers with low education in the intervention group reported to quit at the higher rates than smokers with moderate education in the control group.

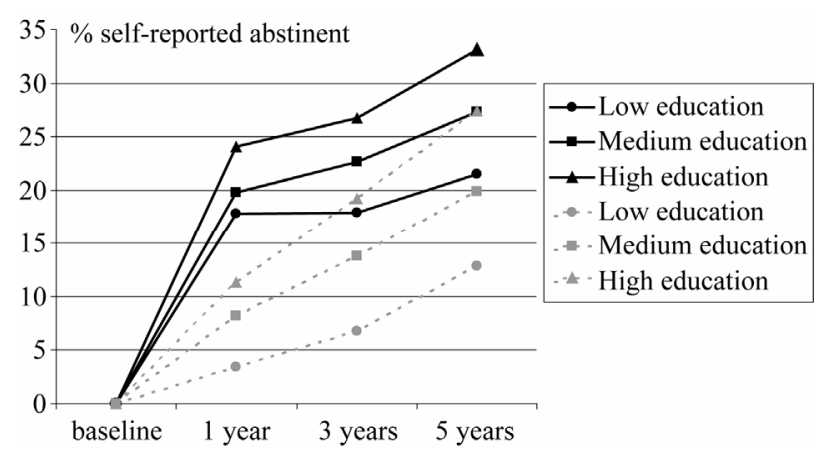

Figure 2. Self-reported abstinence from smoking across socioeconomic groups, measured by length of education. Changes from baseline to 5 years follow-up. High intensity intervention group A (full black lines) compared with control group C (dotted grey lines). The Inter99 study (1999-2006), Copenhagen, Denmark. Generalised linear mixed models under the assumption of missing at random. Included in analyses: Daily smokers at baseline. $\mathrm{N}=1154,1161,1365$ in group $\mathrm{A}$ and 906, 861, 825 in group $\mathrm{C}$, at 1-year, 3-years and 5-years, respectively. 
Table 1. Baseline characteristics of daily smokers in the intervention and the control group of the Inter99 study.

\begin{tabular}{|c|c|c|c|c|c|}
\hline & $\mathrm{N}$ & Intervention group & $\mathrm{N}$ & Control group & p-value \\
\hline Age, years $(\text { mean } \pm \mathrm{SD})^{\#}$ & 1991 & $45.7( \pm 7.8)$ & 1135 & $45.9( \pm 9.6)$ & $<0.001$ \\
\hline $\operatorname{Sex}=\operatorname{man}(\%)$ & 1991 & $49.3 \%$ & 1135 & $49.4 \%$ & 0.955 \\
\hline Smoking spouse $=$ yes & 1793 & $47.2 \%$ & 1010 & $50.5 \%$ & 0.182 \\
\hline Smoking debut, age (mean \pm SD) & 1979 & $17.1( \pm 4.5)$ & 1120 & $17.1( \pm 4.3)$ & 0.703 \\
\hline Pack-years smoked (median, interquartile range) & 1975 & $25.0(19)$ & 1117 & $23.0(19)$ & 0.326 \\
\hline Tobacco consumption, cigarettes/gram pr. day $($ mean $\pm \mathrm{SD})$ & 1984 & $17.7( \pm 8.5)$ & 1129 & $17.3( \pm 8.4)$ & 0.331 \\
\hline Heavy smoker $\geq 15$ cigarettes/gram tobacco pr. day ( $\%)$ & 1129 & $70.5 \%$ & 932 & $69.4 \%$ & 0.516 \\
\hline Ever tried to quit $=$ yes $(\%)$ & 1968 & $73.1 \%$ & 1107 & $71.9 \%$ & 0.414 \\
\hline Number of quit attempts (median, interquartile range) & 1925 & $2.0(3)$ & 1073 & $2.0(3)$ & 0.491 \\
\hline Motivation to quit $=$ preparation stage $(\%)$ & 1847 & $10.7 \%$ & 1042 & $6.9 \%$ & $<0.001$ \\
\hline Employment status = employed $(\%)$ & 1969 & $84.2 \%$ & 1131 & $79.8 \%$ & 0.002 \\
\hline Diet $=$ unhealthy $(\%)$ & 1944 & $22.7 \%$ & 1069 & $28.5 \%$ & 0.001 \\
\hline Physical activity $=$ less active than recommended $(\%)$ & 1858 & $38.5 \%$ & 1045 & $40.9 \%$ & 0.241 \\
\hline Alcohol consumption $=$ above recommendations $(\%)$ & 1911 & $22.9 \%$ & 1100 & $21.6 \%$ & 0.416 \\
\hline Primary, secondary, high school education, years (mean \pm SD) & 1952 & $10.1( \pm 2.3)$ & 1117 & $10.0( \pm 2.5)$ & 0.259 \\
\hline Occupational education (\%) & 1991 & & 1135 & & 0.887 \\
\hline Low education & 464 & $23.3 \%$ & 265 & $23.3 \%$ & \\
\hline Medium education & 1343 & $67.5 \%$ & 771 & $67.9 \%$ & \\
\hline High education & 184 & $9.2 \%$ & 99 & $8.7 \%$ & \\
\hline
\end{tabular}

Bold type: $\mathrm{p}<0.05 ; \mathrm{SD}=$ standard deviation; ${ }^{\#}$ Age groups in the study-population: $30,35,40,45,50,55$ and 60 years. More middle-aged persons were invited in the intervention group than in the control group. In this table we show mean age, but the p-value is shown for the categorical, six-classed, age-variable.

To answer our research-question we looked at the generalised linear mixed model. Odds ratios of abstinence from smoking for persons in the intervention group with low and medium education were generally higher, when comparing with control group, than for persons with high education, even though they were nonsignificant for persons with low education at three and five-year follow-up (Table 2). This indicates that persons with lower SEP apparently benefitted more of the smoking cessation intervention. However, the interaction between group and education was non-significant $(\mathrm{p}=$ $0.510,0.805$ and 0.508 at one-, three- and five-year follow-up), indicating that the differences in quit rates across educational groups were not significantly different in the intervention and the control group. The interaction between education and time was non-significant $(p=$ 0.962 ). Neither did we find the three-way interaction between group, education and time significant $(\mathrm{p}=$ 0.878).

\section{DISCUSSION}

In this randomised population-based intervention study we found that smokers across all educational levels did benefit from the anti-smoking intervention, and that the intervention did not increase the social inequality in smoking. Persons with higher education had generally higher abstinence rates than persons with lower education, both in the intervention and in the control group, and the gap increased over time. Abstinence rates were highest in the intervention group, offering lifestyle counselling and group based smoking cessation, and lowest in the control group.

There is no sign that the intervention had increased the social inequality in smoking. At five-year follow-up, smokers with low education in the intervention group reported to quit at the higher rates than smokers with moderate education in the control group. In general, odds ratios of abstinence from smoking for persons with low/ medium education in the intervention group were higher, than for persons in the control group, indicating that persons with low/medium education maybe benefitted more of the smoking cessation intervention than those with high education. Yet, the interaction term between education and group was not significant. This could be 
Table 2. Probability of being abstinent from smoking across socioeconomic groups, measured by length of education. High intensity intervention group A compared with control group C (= reference). Five years follow-up of the Inter99 study (1999-2006), Denmark.

\begin{tabular}{|c|c|c|c|}
\hline & Low education OR $(95 \% \mathrm{CI})$ & Medium education OR $(95 \% \mathrm{CI})$ & High education OR $(95 \% \mathrm{CI})$ \\
\hline Control group & 1 & 1 & 1 \\
\hline \multicolumn{4}{|l|}{ Intervention group } \\
\hline 1 year & $4.22(1.49-11.9)^{*}$ & $3.02(1.09-4.80)^{* *}$ & $2.65(0.89-7.93)$ \\
\hline 3 years & $2.00(0.88-4.54)$ & $1.81(1.24-2.63)^{* *}$ & $1.06(0.43-2.60)$ \\
\hline 5 years & $2.06(0.99-4.27)$ & $2.71(1.88-3.92)^{* *}$ & $1.62(0.66-3.96)$ \\
\hline
\end{tabular}

explained by the fact that relatively few persons had low and high education (small groups with broad confidence intervals).

To our knowledge, no previous randomised studies have described the effect of a high risk strategy. This strategy, described by Geoffrey Rose, seeks to protect susceptible individuals, and implies some form of screening and risk assessment. This is followed by a prevention practice, for example providing protection against the effects of exposure (e.g. hepatitis vaccine), reducing the level of exposure (e.g. statins) or removing the exposure (e.g. smoking cessation). The high risk strategy is typically implemented in the health care system and is provided by health care professionals [24]. In our study, we screened for high risk of IHD and offered lifestyle counselling in order to remove the negative health effects of an unhealthy lifestyle.

Can we answer the question "what messages and interventions work to get lower socioeconomic groups to stop smoking?" A recent review concluded that there is considerable evidence that media campaigns to promote smoking cessation are often less effective among socioeconomically disadvantaged populations [25]. Increasing the price of cigarettes may provide a means of reducing social disparities in smoking [26,27]. However, not all studies have found this beneficial effect [28], and as smoking prevalence in the Western countries is falling, price increases may become less effective as an inducement for hard-core smokers to quit [29]. A recent study found that a higher price on tobacco actually increased social inequality in smoking, as poor smokers were heavier, more tobacco-dependent smokers, who had difficulties to quit [30].

The effect of a smoking ban in public places, showed a significant reduction in acute coronary events after a smoking ban, which tended to be greater among lower socioeconomic groups [31]. Partial bans, on the other hand (e.g. allowing smoking in some bars/restaurants), may be more likely to worsen socioeconomic inequalities in smoking prevalence [32].

The English National Health Service stop smoking services offer free professional support, and focus on the individual, thereby reminding of the high risk strategy. An observational study found that these services probably make a modest contribution to reducing inequalities in smoking prevalence [33].

It has recently been suggested that a new strategy, "the vulnerable populations theory" could be a good supplement to the other well-established public health strategies. The idea was to move away from the risk factor epidemiological thought, which tends to focus largely on behaviour alone, and suggest that some groups are vulnerable with regard to the social structure and their practices [34]. Other authors have argued that the term "vulnerable populations" is not without problems, including potential stigmatisation [35]. It is important that we debate the consequences of the established public health strategies and discuss new strategies.

This study shows that it is feasible to attract smokers with low SEP to a programme with lifestyle counselling, and group-based smoking cessation. The Inter99 study used a proactive recruitment strategy by sending personal invitations with a prearranged date and time. It is worth to note that smokers with low education who were offered assistance to quit obtained higher quit-rates than smokers with moderate education in the control group, at five-year follow-up.

The multi-factorial approach (the lifestyle consultation also addressed diet, physical activity and alcohol consumption) may have been important in order to reach a more unselected group of smokers. Probably, many smokers, and especially smokers with low education, would not have attended the clinic if we had invited smokers only, and focused on smoking cessation only. Successful change of lifestyle inspired some non-motivated smokers. e.g. we experienced that some obese smokers, who had experienced a successful loss of weight when participating in a diet and exercise group, found confidence to join a smoking cessation group at next follow-up visit. Thus, the potentially active components of this intervention are difficult to untangle.

Measuring SEP is very complex and each measure- 
ment has different strengths and weaknesses. There is no single best indicator of SEP [36,37]. In general, education is relevant to people regardless of age or working circumstances, it is a strong determinant of employment and income and it reflects knowledge. The variable used in this paper, occupational education, is a frequently used measure of SEP in Denmark, significantly associated with chronic disease [19].

The most important weakness is the low participation rate and drop-out in the follow-up, which leads to selection, as abstinence is known to be associated with attendance [38]. In a baseline publication we found that participation rate was higher in younger women than in younger men, and it increased with increasing age until 55 years of age after which it declined. The participants in the intervention group did not differ from those in the control group, regarding former admissions for all causes, IHD, CVD, and diabetes [14]. We suppose that there was a higher dropout in continuous smokers in the intervention, as the expectations of quitting were higher than in the control group. The social non-smoking norm in those with high education would probably result in a higher dropout of continuous smokers with high education. We can not rule out the possibility that bias in self-report may have affected results. In both cases we suppose that bias would be more pronounced in the intervention groups than in the control group. The possibility of residual confounding due to unknown or unmeasured confounders always exists.

The strengths of this study are the randomisation, the large size, the long follow-up and the setting in a general population, including unselected smokers from all socioeconomic levels. The known baseline differences between smokers in the intervention and the control group have been adjusted for. The use of advanced statistical analyses which hold under a missing at random assumption increases the probability of valid results. Even though we only presented self-reported abstinence in this paper, smoking abstinence has previously been validated in the intervention groups [18].

\section{Conclusion}

In this population-based randomised intervention study, smokers across all educational levels benefited from the anti-smoking intervention, and the intervention did not increase the social inequality in smoking. It is worth to note that smokers with low education who were offered assistance to quit obtained as high quit-rates as smokers with moderate/high education in the control group, at five-year follow-up. As there is evidence that mass media increase social inequality in smoking, legislation combined with proactive smoking cessation support to smokers with low SEP seem a better alternative, when planning strategies to reduce social inequalities in health.

\section{ACKNOWLEDGEMENTS}

We thank the whole Inter99-staff and all persons participating in the study. Also, we thank the funding providers. Both those who funded this paper and those who funded the Inter99 study. This work was supported by: Tryg Foundation and Helse Foundation. The Inter99 study was funded by: Danish Medical Research Council; The Danish Centre for Evaluation and Health Technology AsSEPsment; Novo Nordisk; Copenhagen County; Danish Heart Foundation; The Danish Pharmaceutical Association; Augustinus Foundation; Becket Foundation; Ib Henriksens Foundation. The researchers are all independent of the founders. The study was initiated by Torben Jorgensen, Knut Borch-Johnsen, Troels Thomsen and Hans Ibsen. The Steering Committee of the Inter99 study: Professor D.M.Sci. Torben Jorgensen (principal investigator), Professor D.M.Sci., Knut Borch-Johnsen (principal investigator on the diabetes part) and Ph.D. MPH Charlotta Pisinger.

\section{REFERENCES}

[1] Lundberg, O. (1991) Causal explanations for class inequality in health-An empirical analysis. Social Science \& Medicine, 32, 385-393.

doi:10.1016/0277-9536(91)90339-E

[2] Marmot, M. (2005) The social environment and health. Clinical Medicine, 5, 244-248.

[3] Wagstaff, A. and van Doorslaer, E. (2000) Income inequality and health, what does the literature tell us? Annual Review of Public Health, 21, 543-567.

doi:10.1146/annurev.publhealth.21.1.543

[4] Gruer, L., Hart, C.L., Gordon, D.S. and Watt, G.C. (2009) Effect of tobacco smoking on survival of men and women by social position: A 28 year cohort study. British Medical Journal, 338, b480. doi:10.1136/bmj.b480

[5] Borrell, C., Rue, M., Pasarin, M.I., et al. (2000) Trends in social class inequalities in health status, health-related behaviors, and health services utilization in a Southern European urban area (1983-1994). Preventive Medicine, 31, 691-701. doi:10.1006/pmed.2000.0751

[6] Giskes, K., Kunst, A.E., Benach, J., et al. (2005) Trends in smoking behaviour between 1985 and 2000 in nine European countries by education. Journal of Epidemiology \& Community Health, 59, 395-401. doi:10.1136/jech.2004.025684

[7] Osler, M., Gerdes, L.U., Davidsen, M., et al. (2000) Socioeconomic status and trends in risk factors for cardiovascular diseases in the Danish MONICA population, 1982-1992. Journal of Epidemiology \& Community Health, 54, 108-113. doi:10.1136/jech.54.2.108

[8] Osler, M., Prescott, E., Gottschau, A., et al. (1998) Trends in smoking prevalence in Danish adults, 1964-1994. The influence of gender, age, and education. Scandinavian Journal of Social Medicine, 26, 293-298. doi:10.1177/14034948980260041101

[9] Rasmussen, M., Due, P., Damsgaard, M.T. and Holstein, B.E. (2009) Social inequality in adolescent daily smoking, 
has it changed over time? Scandinavian Journal of Public Health, 37, 287-294. doi:10.1177/1403494809102178

[10] Wanless, D. and Treasury, H.M. (2004) Securing good health for the whole population, Final report 2004.

[11] Pisinger, C., Vestbo, J., Borch-Johnsen, K. and Jorgensen, T. (2005) Smoking cessation intervention in a large randomised population-based study. The Inter99 study. Preventive Medicine, 40, 285-292. doi:10.1016/j.ypmed.2004.06.001

[12] Aadahl, M., von Huth, S.L., Toft, U., Pisinger, C. and Jorgensen, T. (2009) Does a population-based multi-factorial lifstyle intervention increase social inequality in physical activity? The Inter99 study. British Journal of Sports Medicine, 45, 209-215. doi:10.1136/bjsm.2009.064840

[13] Toft, U., Jakobsen, M., Aadahl, M., Pisinger, C. and Jorgensen, T. (2011) Does a population-based multi-factorial lifestyle intervention increase social inequality in dietary habits? The Inter99 study. Preventive Medicine, 54, 8893. doi:10.1016/j.ypmed.2011.10.005

[14] Jorgensen, T., Borch-Johnsen, K., Thomsen, T.F., et al. (2003) A randomized non-pharmacological intervention study for prevention of ischaemic heart disease, baseline results Inter99. European Journal of Preventive Cardiology, 10, 377-386. doi:10.1097/01.hjr.0000096541.30533.82

[15] The Inter99 Steering Committee, Homepage of the Inter99 study, 2008. www.Inter99.dk

[16] Pisinger, C., Vestbo, J., Borch-Johnsen, K. and Jorgensen, T. (2005) It is possible to help smokers in early motivetional stages to quit. The Inter99 study. Preventive Medicine, 40, 278-284. doi:10.1016/S0091-7435(04)00317-2

[17] Pisinger, C., Vestbo, J., Borch-Johnsen, K., Thomsen, T. and Jorgensen, T. (2005) Acceptance of the smoking cessation intervention in a large population-based study, the Inter99 study. Scandinavian Journal of Public Health, 33, 138-145. doi:10.1080/14034940410028370

[18] Pisinger, C., Glumer, C., Toft, U., et al. (2008) High risk strategy in smoking cessation is feasible on a population-based level. The Inter99 study. Preventive Medicine, 46, 579-584. doi:10.1016/j.ypmed.2008.02.026

[19] Avlund, K., Holstein, B.E., Osler, M., et al. (2003) Social position and health in old age, the relevance of different indicators of social position. Scandinavian Journal of Public Health, 31, 126-136. doi:10.1080/14034940210134130

[20] DiClemente, C.C., Prochaska, J.O., Fairhurst, S.K., et al. (1991) The process of smoking cessation, an analysis of precontemplation, contemplation, and preparation stages of change. Journal of Consulting and Clinical Psychology, 59, 295-304. doi:10.1037/0022-006X.59.2.295

[21] Prochaska, J.O. and DiClemente, C.C. (1983) Stages and processes of self-change of smoking, toward an integrative model of change. Journal of Consulting and Clinical Psychology, 51, 390-395. doi:10.1037/0022-006X.51.3.390

[22] Toft, U., Kristoffersen, L.H., Lau, C., Borch-Johnsen, K. and Jorgensen, T. (2007) The dietary quality score, vali- dation and association with cardiovascular risk factors, the Inter99 study. European Journal of Clinical Nutrition, 61, 270-278. doi:10.1038/sj.ejen.1602503

[23] Von Huth, S.L., Borch-Johnsen, K. and Jorgensen, T. (2007) Commuting physical activity is favourably associated with biological risk factors for cardiovascular disease. European Journal of Epidemiology, 22, 771-779. doi:10.1007/s10654-007-9177-3

[24] Rose, G. (2001) Sick individuals and sick populations. International Journal of Epidemiology, 30, 427-432. doi:10.1093/ije/30.3.427

[25] Niederdeppe, J., Kuang, X., Crock, B. and Skelton, A. (2008) Media campaigns to promote smoking cessation among socioeconomically disadvantaged populations, what do we know, what do we need to learn, and what should we do now? Social Science \& Medicine, 67, 1343-1355. doi:10.1016/j.socscimed.2008.06.037

[26] Siahpush, M., Wakefield, M.A., Spittal, M.J., Durkin, S.J. and Scollo, M.M. (2009) Taxation reduces social disparities in adult smoking prevalence. American Journal of Preventive Medicine, 36, 285-291. doi:10.1016/j.amepre.2008.11.013

[27] Townsend, J., Roderick, P. and Cooper, J. (1994) Cigarette smoking by socioeconomic group, sex, and age, effects of price, income, and health publicity. British Medical Journal, 309, 923-927. doi:10.1136/bmj.309.6959.923

[28] Khang, Y.H., Yun, S.C., Cho, H.J. and Jung-Choi, K. (2009) The impact of governmental antismoking policy on socioeconomic disparities in cigarette smoking in South Korea. Nicotine \& Tobacco Research, 11, 262-269. doi:10.1093/ntr/ntn036

[29] Sheu, M.L., Hu, T.W., Keeler, T.E., Ong, M. and Sung, H.Y. (2004) The effect of a major cigarette price change on smoking behavior in california, a zero-inflated negative binomial model. Health Economics, 13, 781-791. doi:10.1002/hec.849

[30] Peretti-Watel, P. and Constance, J. (2009) It's all we got left. Why poor smokers are less sensitive to cigarette price increases. International Journal of Environmental Research and Public Health, 6, 608-621. doi:10.3390/ijerph6020608

[31] Cesaroni, G., Forastiere, F., Agabiti, N., et al. (2008) Effect of the Italian smoking ban on population rates of acute coronary events. Circulation, 117, 1183-1188. doi:10.1161/CIRCULATIONAHA.107.729889

[32] Woodall, A.A., Sandbach, E.J., Woodward, C.M., Aveyard, P. and Merrington, G. (2005) The partial smoking ban in licensed establishments and health inequalities in England, modelling study. British Medical Journal, 331, 488-489. doi:10.1136/bmj.38576.467292.EB

[33] Bauld, L., Judge, K. and Platt, S. (2007) Assessing the impact of smoking cessation services on reducing health inequalities in England, observational study. Tobacco Control, 16, 400-404. doi:10.1136/tc.2007.021626

[34] Frohlich, K.L. and Potvin, L. (2008) Transcending the known in public health practice, the inequality paradox, the population approach and vulnerable populations. American Journal of Public Health, 98, 216-221. 


\section{doi:10.2105/AJPH.2007.114777}

[35] McLaren, L., McIntyre, L. and Kirkpatrick, S. (2010) Rose's population strategy of prevention need not increase social inequalities in health. International Journal of Epidemiology, 39, 372-327. doi:10.1093/ije/dyp315

[36] Galobardes, B., Shaw, M., Lawlor, D.A., Lynch, J.W. and Davey, S.G. (2006) Indicators of socioeconomic position (part 1). Journal of Epidemiology \& Community Health, 60, 7-12. doi:10.1136/jech.2004.023531
[37] Galobardes, B., Shaw, M., Lawlor, D.A., Lynch, J.W. and Davey, S.G. (2006) Indicators of socioeconomic position (part 2). Journal of Epidemiology \& Community Health, 60, 95-101. doi:10.1136/jech.2004.028092

[38] Patterson, F., Jepson, C., Kaufmann, V., et al. (2003) Predictors of attendance in a randomized clinical trial of nicotine replacement therapy with behavioral counseling. Drug and Alcohol Dependence, 72, 123-131. doi:10.1016/S0376-8716(03)00194-7 